\title{
The Results of a Long-Term Follow-up of Bilateral Single Port Sympathicotomy in Primary Hyperhidrosis: Should We Per- form This Surgery?
}

\author{
Timuçin ALAR ${ }^{\mathrm{a}^{*}}$, İsmail Ertuğrul GEDİK ${ }^{\mathrm{a}}$ \\ ${ }^{a}$ Department of Thoracic Surgery, Çanakkale Onsekiz Mart University Medical Faculty, Çanakkale, 17100, Turkey.
}

\begin{abstract}
Background: Hyperhidrosis $(\mathrm{HH})$, which refers to excessive sweating of the body in response to temperature or emotional stimuli rather than physiological stimuli, can adversely affect quality of life. In this prospective study, we investigated the long-term effects, development of complications, and patient satisfaction among those who underwent bilateral single-port endoscopic thoracic sympathecotomy (ETS) for HH.

Methods: Thirty-one patients who underwent bilateral single-port endoscopic thoracic sympathicotomy (ETS) for HH between January 200 and November 2014 were enrolled in this study. The patients wer1e followed up until July 2017. Patient satisfaction in terms of the primary complaint (PC) and complications, such as compensatory hyperhidrosis $(\mathrm{CH})$, in the short and long term were recorded.

Results: The mean follow-up period was $60.6 \pm 12.8$ (min: 40, max: 89) mo postoperatively. When both the short- and long-term results were evaluated together, $\mathrm{CH}$ had no effect on patient satisfaction. However, persistence of the PC in both the short $(p=0.020)$ and long term $(p=0.001)$ had a significant effect on satisfaction. Conclusion: The most important factor affecting patient satisfaction was PC persistence. Thus, further studies should be performed to enlighten this complication. even though it may remit with time. Whether ETS is a permanent treatment that cannot successfully treat PC, possible complications and the ability to cope with them will seem to be open to debate as the most important issues that surgeons will have to face in the near future. Keywords: Complication; hyperhidrosis; surgery; sympathicotomy
\end{abstract}

\section{INTRODUCTION}

Hyperhidrosis $(\mathrm{HH})$ can be described as excessive sweating of the body that exceeds physiological needs ${ }^{[1]}$. Hyperhidrosis may be primary or secondary. Primary $\mathrm{HH}$ is the result of to overactivity of the sympathetic nervous system, whereas secondary HH is caused by various factors, such as malignancies, endocrine disorders (e.g., thyrotoxicosis), and some medications ${ }^{[1-3]}$. Primary HH affects facial, palmar, axillary, and plantar regions of the body, exhibiting regional and symmetrical involvement patterns due to sympathetic nervous system overactivity ${ }^{[1,2]}$.

Hyperhidrosis may be treated via medical or surgical ap-

*Corresponding author: Timuçin ALAR

Mailing address: Department of Thoracic Surgery, Çanakkale Onsekiz Mart University Medical Faculty, Çanakkale Onsekiz Mart Universitesi Tıp Fakultesi Gogus Cerrahisi Anabilim Dalı Terzioglu Girisi, Çanakkale,17100,Turkey.

E-mail: timalar@yahoo.com

Received: 24 October 2019 Accepted: 25 November 2019 proaches. Endoscopic thoracic sympathecotomy (ETS) is the most commonly used type of surgical treatment today. The surgery can be performed in a variety of ways, such as dissecting the sympathetic chain in the thoracic region, thermal damage via electrocautery, or clipping. Among various ETS-related complications, which include a hemo-pneumothorax, Horner's syndrome, and bradycardia, the mostimportant in terms of both patient and physician satisfaction is compensatory hyperhidrosis $(\mathrm{CH})^{[4,5]}$.

In this prospective study, we investigated the rate of complications, such as $\mathrm{CH}$, and $\mathrm{PC}$ persistence in the long-term postoperative period following bilateral single port ETS and the effects of these complications on patient satisfaction.

\section{METHODS}

Patients who had undergone single port ETS between January 2010 and November 2014 at Çanakkale Onsekiz Mart University Medical Faculty Thoracic Surgery Clinic were prospectively followed up until July 2017. During outpatient clinical examinations and telephone inter- 
views, the patients were questioned about changes in $\mathrm{CH}$ and their PC in the postoperative period and their satisfaction with the operation.

In the 6 mo prior to the single port ETS, all the patients had received medical treatment. Despite this treatment, none of the patients experienced improvements in their PC. Prior to surgery, informed consent was obtained from each patient. In patients with $\mathrm{HH}$ affecting multiple areas, only the area most affected was treated. The ETS was performed using a 10-mm 0-degree thoracoscope (Karl Storz $26034 \mathrm{AA}$ ) with a 6-mm working channel under general anesthesia via a double-lumen endotracheal tube. After the patient had been positioned in the lateral decubitus position, a single thoracoport (width: $10.5 \mathrm{~mm}$ ) was inserted into the thorax through a single incision of $11 \mathrm{~mm}$ at the intersection of the fourth intercostal space and mid-axillary line (Figure 1). The sympathetic chain was cauterized by endoscopic electrocautery from the T2 for fascial hyperhidrosis, T3 for palmar hyperhidrosis, T4 for axillary hyperhidrosis, and $\mathrm{T} 5$ for plantar hyperhidrosis. In addition, during the sympathecotomy of the T2-T3, the presence of the nerve of Kuntz was verified, and the nerve tissue was cauterized. The rib bed was cauterized laterally for an additional $2 \mathrm{~cm}$ to allow the dissection of possible bypassing nerve fibers. After hemorrhage control, the air in the pleural cavity was evacuated via a small catheter. The incision line was closed by subcutaneous suturing, and the same procedure was repeated on the other side. On the postoperative first day, the patients were evaluated by a physical examination, posterior-anterior chest X-ray, hemogram, and blood biochemistry tests. Patients whose physical examination and examination results revealed no abnormalities were discharged from the hospital on the first postoperative day. The patients were referred to the outpatient clinic on the seventh postoperative day and reevaluated via a physical examination and posterior-anterior chest X-ray. In the third postoperative month and again in the third postoperative year, all the patients were interviewed by telephone. They were questioned about their $\mathrm{HH}$ status, $\mathrm{CH}$ status, and satisfaction with the operation. They were asked to rate their $\mathrm{HH}$ at the site of the $\mathrm{PC}$ from 0 to 10 , where 0 represented no sweating, and 10 represented the most severe sweating.

The follow-up times were from the date of the operation to the last follow-up date (July 2017), expressed in months. Subsequently, cutoff values at 48,60 , and 72 mo were coded as a dichotomous variable for those below and above the follow-up period. The following parameters were coded as dichotomous variables: $\mathrm{CH}, \mathrm{PC}$, and patient satisfaction. In terms of the latter, on the day of the interview, the patients were asked whether, given the choice, they would undergo the surgery again. The

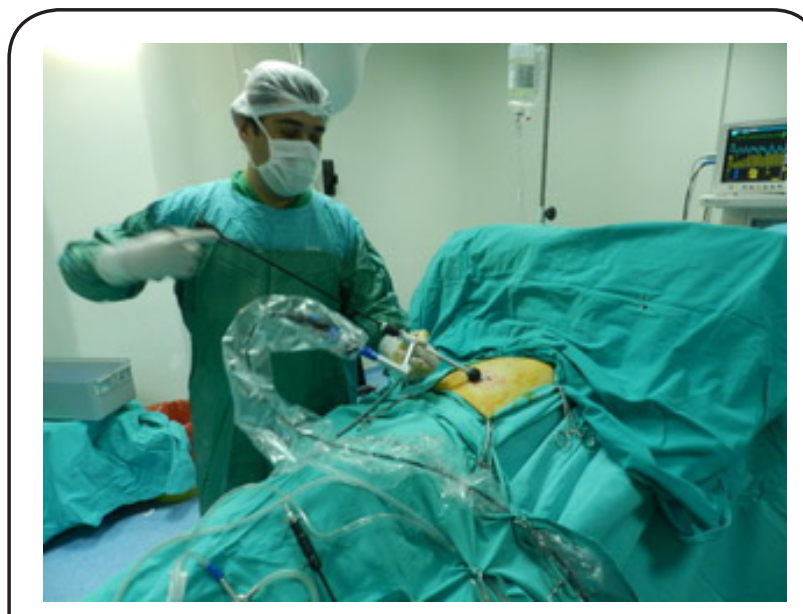

Figure 1. Thoracoscope Insertion Through Single Port Incision.

CH and PC intensities were coded as continuous variables ranging from 1 to 10 .

The data were then transferred to a digital medium. The normal distribution matching of the continuous variables was examined for the frequency and distribution of variables. The relations between the continuous variables were analyzed using appropriate correlation tests, and the relations between the dichotomous variables were analyzed using a chi-square test and, if necessary, Fisher's exact test. In all the analyses, statistical significance was accepted as a p-value of $<0.05$. The absolute $\mathrm{p}$ values are given for the analysis results.

\section{RESULTS}

Thirty-one patients who underwent bilateral single port ETS at Çanakkale Onsekiz Mart University Medical Faculty Chest Surgery Clinic between January 2010 and November 2014 were included in the study. Sixteen (51.6\%) of the patients were males, and 15 (48.4\%) were females. The mean age of the patients was $24.13 \pm$ 4.64 (min: 13, max: 34) y. In terms of the site of the PC, it was craniofacial in $6.5 \%(\mathrm{n}=2)$ of cases, palmar-facial in $12.9 \%(n=4)$ of cases, palmar in $67.7 \%(n=21)$ of cases, axillary in $9.7 \%(n=3)$ of cases, and palmoplantar in $3.2 \%(n=1)$ of cases.

The postoperative complications were a pneumothorax in one patient and a hemopneumothorax in one patient. Tube thoracostomy was performed in the patient who developed a hemopneumothorax, and air evacuation via thoracentesis was performed in the other patient. All the other patients were discharged on the first postoperative day. Thus, the average duration of the hospital stay was $1.13 \pm 0.56$ (min: 1 , max: 4 ) d. The mean follow-up period was $60.6 \pm 12.8$ (min: 40, max: 89) mo postoperatively.

When the patients were questioned about their PC status, $10(32.3 \%)$ of the 31 patients responded that their 
Table 1. Regional Distribution of Ongoing Hyperhidroses.

\begin{tabular}{lcc}
\hline Region & n & \% \\
\hline Craniofacial & 1 & 10 \\
Palmar & 5 & 50 \\
Axillary & 3 & 30 \\
Plantar & 1 & 10 \\
Total & 10 & 100 \\
\hline
\end{tabular}

Table 2. The Distribution of the Severity of Compensatory Hyperhidrosis.

\begin{tabular}{lcc}
\hline Severity & $\mathbf{n}$ & $\mathbf{\%}$ \\
\hline 3 & 1 & 6.8 \\
4 & 2 & 13.3 \\
5 & 2 & 13.3 \\
6 & 2 & 13.3 \\
7 & 3 & 20 \\
8 & 3 & 20 \\
10 & 2 & 13.3 \\
Total & 15 & 100 \\
\hline
\end{tabular}

Table 3. The Regional Distribution of Compensatory Hyperhidrosis.

\begin{tabular}{lcc}
\hline Location & $\mathbf{n}$ & \% \\
\hline Dorsal & 9 & 60 \\
Abdomen & 3 & 20 \\
Chest & 3 & 20 \\
Total & 15 & 100 \\
\hline
\end{tabular}

PC was not resolved. The remaining 21 cases responded that their PC was in remission. Among patients with a persistent $\mathrm{PC}$, the most frequent site was palmar $\mathrm{HH}$ (Table 1).

When the patients were asked about their $\mathrm{CH}$ status in the postoperative third-month, $15(48.4 \%)$ of the 31 patients reported that they had developed $\mathrm{CH}$. When questioned about the severity of the $\mathrm{CH}$, the severity ranged from 3-10, with the most frequent $\mathrm{CH}$ site being the dorsal region (Tables 2 and 3).

At the time of the final interview in July 2017, the patients were questioned about their satisfaction with the surgery. Six (19\%) of the 31 patients were dissatisfied with the surgery, and $81 \%$ of patients were satisfied. Five of the six (83\%) patients who were not satisfied with the surgery had developed $\mathrm{CH}$, and the $\mathrm{PC}$ was unresolved in five of these six (83\%) cases (Tables 4,5$)$. In total, seven patients indicated that if given the choice today, they would not have the surgery. Two of these seven patients developed $\mathrm{CH}$ postsurgery, and the PC was unresolved in the other five cases. One patient who was satisfied with the surgery reported that he would not undergo this surgery today. This patient had some relief from his $\mathrm{PC}$, but it was not completely resolved.
Table 4. Primary Complaint versus a Satisfaction Cross Table.

\begin{tabular}{lccc}
\hline & \multicolumn{2}{c}{ Primary complaint } \\
\cline { 2 - 4 } & - & + & Total \\
\hline Satisfaction & 1 & 5 & 6 \\
No & 20 & 5 & 25 \\
Yes & 21 & 10 & 31 \\
Total & 21 & \\
\hline
\end{tabular}

${ }^{*} p>0.05$, Fisher's exact test value 0.007.

Table 5. Compensatory Hyperhidrosis versus a Satisfaction.

\begin{tabular}{lccc}
\hline & \multicolumn{2}{c}{ Compensatory Hyperhidrosis } \\
\cline { 2 - 4 } & - & + & Total \\
\hline Satisfaction & 1 & 5 & 6 \\
No & 15 & 10 & 25 \\
Yes & 16 & 15 & 31 \\
Total & & & \\
\hline
\end{tabular}

${ }^{*} p>0.05$, Fisher's exact test value 0.083 .

When the long-term follow-up of the cases was examined, the PC was resolved in two patients, and $\mathrm{CH}$ developed in three patients. In total, there were $8(25 \%)$ cases of PC persistence and 18 (58\%) cases of $\mathrm{CH}$ persistence. Persistence of the $\mathrm{PC}$ affected patient satisfaction in both the short $(p=0.007)$ and long term $(p=0.001)$. In contrast, $\mathrm{CH}$ had no effect on patient satisfaction in either the short $(\mathrm{p}=0.083)$ or long term ( $\mathrm{p}=0.359)$.

\section{DISCUSSION}

Although the precise pathophysiological mechanism in $\mathrm{HH}$ is not known, excessive stimulation of thermoregulatory and emotional sweating control centers or excessive secretion of acetylcholine by the sympathetic nervous system in response to stimulation of these centers may play a role ${ }^{[3]}$. Individuals with primary $\mathrm{HH}$, which usually begins in childhood or adolescence, generally consult a physician because of psychosocial problems caused by $\mathrm{HH}^{[6]}$.

Medical therapy options for HH include topical or systemic antiperspirants, iontophoresis, botulinum toxin injections, laser therapy, and microwave therapy ${ }^{[6]}$. Topical antiperspirant agents consist of a $20 \%$ solution of aluminum hydrochloride ${ }^{[7]}$. Topical and systemic forms of anticholinergics include glycopyrrolate and oxybutynin ${ }^{[7]}$. Iontophoresis is the treatment of choice for palmoplantar HH. The treatment is based on the principle that the palmoplantar zone is kept in tap water in which a low voltage electrical current is applied for about $30 \mathrm{~min}{ }^{[8,9]}$. Botulinum toxin A injections are used as a treatment for both palmoplantar and axillary $\mathrm{HH}$. These provide a longer remission time than any other medical treatment, with remission of $\mathrm{HH}$ for about 6 mo 
post-treatment ${ }^{[10]}$. In terms of laser treatment, a neodynium yttrium aluminum garnet laser is used for $\mathrm{HH}^{[11]}$. In microwave therapy, thermolysis of eccrine sweat glands is conducted. The main difference of this method is that it is irreversible ${ }^{[12]}$. The main disadvantage of medical treatments for $\mathrm{HH}$ as compared to surgical treatment is that medical therapy offers only a temporary solution to an ongoing problem, and the treatments have to be repeated. An important advantage of medical therapy versus surgical treatment is the absence of $\mathrm{CH}$, which is a complication of surgery. For this reason, all $\mathrm{HH}$ patients are advised to receive medical treatment for at least 6 mo before considering surgery. If the medical treatment is unsuccessful, with success defined as patient satisfaction, surgery is an option. In the present study, none of the patients had satisfactory outcomes prior to undergoing surgery.

$\mathrm{CH}$, a complication of ETS, refers to excessive sweating affecting various parts of the bodys. The mechanism underlying the development $\mathrm{CH}$ is not known. Although some authors have argued that this condition develops because of negative feedback stimuli of the sympathetic chain cannot reach the hypothalamus, there is no scientific evidence to support this hypothesis ${ }^{[13,14]}$. Approximately 3-98\% of patients develop CH after ETS, and it is most commonly observed in the trunk area. It is not possible to predict who may develop $\mathrm{CH}$ and how severe it may become ${ }^{[4,5,13,15]}$. Although some studies showed that a sympathecotomy at the $\mathrm{T} 2$ level or a sympathectomy at multiple levels and older age increased the risk of developing $\mathrm{CH}$, other studies contradicted these findings ${ }^{[13,16-18]}$. In our study, in the short term, the rate of $\mathrm{CH}$ was $48 \%$, whereas it was $58 \%$ in the long term. $\mathrm{CH}$ developed most commonly in the trunk region, similar to the scientific literature on this subject.

Previous research reported that the development of $\mathrm{CH}$ and PC persistence were the two most important factors affecting patient satisfaction after ETS operations. In the literature, the reported rates of PC persistence range from 0 to $21.4 \%{ }^{[13,17,19]}$. The rates in the present study were comparable to those found in the literature, with rates of $32 \%$ in the short-term follow up and $25 \%$ in the long-term follow up. An interesting finding in the present study was that PC persistence was the most important factor affecting patient satisfaction in the long term.

In previous studies on $\mathrm{HH}$, the postoperative follow-up period was less than $1 \mathrm{y}$, with most follow-ups lasting approximately 6 mo. Thus, knowledge is limited on the status of $\mathrm{HH}$ among these patients over the long term. In the present study, the patients were followed up for a mean of 60 mo. As shown by the results, an increase in the rate of $\mathrm{CH}$ did not increase patient satisfaction. In contrast, while a decrease in PC was observed, there was also a decrease in the patient satisfaction. Thus, the most important factor affecting patient satisfaction was continuation of the PC in contrast to our current knowledge. According to a previous study, the results of intermittent unilateral ETS for redu cing $\mathrm{CH}$, which affects patient satisfaction in the short term, are promising, although the duration of the follow-up in the study was only $1 \mathrm{y}^{[20]}$. However, the hospital costs associated with intermittent unilateral ETS seem to be disadvantages, with the procedure requiring two surgeries, two anesthesia sessions, and two hospitalizations. We believe that the primary goal of surgery should be complete resolution of the PC. Self-remission of the PC with age should also be seen as a disadvantage for the ETS ${ }^{[21]}$. The possibility that the $\mathrm{PC}$ may regress in time, combined with the fact that PC persistence rather than $\mathrm{CH}$ affects patient satisfaction, casts doubts on the feasibility of ETS for HH.

The present study has some limitations. The number of cases $(\mathrm{N}=31)$ may be considered low as compared with that in other studies on ETS. In addition, we did compare the ETS method with medical treatment methods in terms of patient satisfaction. Furthermore, we did not use standardized questionnaires to assess quality of life and patient satisfaction with ETS. Thus, the answers we obtained in our interviews may be considered "subjective." As with all forms of "satisfaction," the term "patient satisfaction" is subjective. The development of standardized questionnaires on quality of life and patient satisfaction with ETS would improve the objectivity of patient responses.

\section{CONCLUSION}

For HH, ETS is the only treatment that has been proven to be successful in the long term. The two most important factors affecting patient satisfaction after ETS are the continuation of the PC and $\mathrm{CH}$. Intermittent unilateral ETS may be considered an alternative surgical approach for $\mathrm{CH}$, although the likelihood of $\mathrm{CH}$ may increase in the long term. Furthermore, the PC of HH may regress with age. This treatment should be described as temporary (at least until the end of the adolescence period) rather than permanent. The decision whether to perform this surgery is up to the surgeon. However, the decision whether to be treated with surgery for $\mathrm{HH}$ should be the patients after all these data have been conveyed.

\section{DECLARATIONS}

\section{Authors' contributions}

Made substantial contributions to conception and design of the study and performed data analysis and interpretation: Alar T and Gedik İ E.

Performed data acquisition, as well as provided administrative, technical, and material support: Alar $\mathrm{T}$ and 
Gedik İ E.

\section{Conflicts of interest}

All authors declare that there are no conflicts of interest.

\section{REFERENCES}

1. Smith, F. C. T. (2013). Hyperhidrosis. Surgery, 31,251-255

2. Stefaniak, T. J., and Ćwigoń, M. (2013). Long-term results of thoracic sympathectomy for primary hyperhidrosis. Pol Przegl Chir, 85,247-252

3. Stashak, A-B., Brewer, J. D. (2014). Management of Hyperhidrosis. Clinical, Cosmetic and Investigational Dermatology, 7,285-299

4. Drott, C., Gothberg, G., and Claes, G. (1995). Endoscopic transthoracic sympathectomy: an efficient and safe method for the treatment of hyperhidrosis. J Am Acad Dermatol, 33,78-78

5. Chwajol, M., Barrenechea, I. J., Chakraborty, S., Lesser, J. B., Connery, C. P., and Perin, N. I. (2009). Impact of compensatory hyperhidrosis on patient satisfaction after endoscopic thoracic sympathectomy. Neurosurgery, 64,511-518

6. Brown, A. L., Gordon, J., and Hill, S. (2014). Hyperhidrosis: review of recent advances and new therapeutic options for primary hyperhidrosis. Curr Opin Pediatr, 26,460-465

7. Wozniacki, L., and Zubilewicz, T. (2009). Primary hyperhidrosis controlled with oxybutynin after unsuccessful surgical treatment. Clin Exp Dermatol, 34,e990-991

8. Ozcan, D., and Güleç, A. T. (2014). Compliance with tap water iontophoresis in patients with palmoplantar hyperhidrosis. J Cutan Med Surg, 18,109-113

9. Siah, T. W., and Hampton, P. J. (2013). The effectiveness of tap water iontophoresis for palmoplantar hyperhidrosis using a Monday, Wednesday, and Friday treatment regime. Dermatol Online J, 19,14

10. Lecouflet, M., Leux, C., Fenot, M., Célerier, P., and Maillard, H. (2014). Duration of efficacy increases with the repetition of botulinum toxin A injections in primary palmar hyperhidrosis: A study of 28 patients. J Am Acad Dermatol, 70,1083-1087

11. Letada, P. R., Landers, J. T., Uebelhoer, N. S., and Shumaker, P. R. (2012). Treatment of focal axillary hyperhidrosis using a long pulsed Nd: YAG $1064 \mathrm{~nm}$ laser at hair reduction settings. J Drugs Dermatol, 11,59-63
12. Hong, H. C., Lupin, M., O’Shaughnessy, K. F. (2012). Clinical evaluation of a microwave device for treating axillary hyperhidrosis. Dermatol Surg, 38,728-735

13. Cai, S., Huang, S., An, J., Li, Y., Weng, Y., Liao, H., Chen, H., Liu, L., He, J., and Zhang, J. (2014). Effect of lowering or restricting sympathectomy levels on compensatory sweating. Clin Auton Res, 24,143-149

14. Chou, S. H., Kao, E. L., Lin, C. C., Chang, Y. T., Huang, M. F. (2006). The importance of classification in sympathetic surgery and a proposed mechanism for compensatory hyperhidrosis: experience with 464 cases. Surg Endosc, 20,1749-1753.

15. Waseem, M, Hajjar., Sami, A, Al-Nassar., Heba, M, Al-Sharif., Dana, M. Al-Olayet., Wejdan, S, Al-Otiebi., Alanoud, A, Al-Huqayl., Adnan, W, Hajjar. (2019). The quality of life and satisfaction rate of patients with upper limb hyperhidrosis before and after bilateral endoscopic thoracic sympathectomy Saudi J Anaesth, 13,16-22.

16. Baumgartner, F. J., Reyes, M., Sarkisyan, G. G., Iglesias, A., Reyes, E. (2011). Thoracoscopic sympathicotomy for disabling palmar hyperhidrosis: a prospective randomized comparison between two levels. Ann Thorac Surg, 92,2015-2019.

17. Aoki, H., Sakai, T., Murata, H., Sumikawa, K. (2014). Extent of sympathectomy affects postoperative compensatory sweating and satisfaction in patients with palmar hyperhidrosis. J Anesth, 28,210-213.

18. Licht PB, Pilegaard HK. (2004). Severity of compensatory sweating after thoracoscopic sympathectomy. Ann Thorac Surg, 78,427-431.

19. Delaplace, M., Dumont, P., Lorette, G., Machet, L., Lagier, L., Maruani, A., Samimi, M. (2015). Factors associated with long-term outcome of endoscopic thoracic sympathectomy for palmar hyperhidrosis: a questionnaire survey in a cohort of French patients. Br J Dermatol, 172,805-807

20. Youssef, T., Soliman, M. (2015). Unilateral Sequential Endoscopic Thoracic Sympathectomy for Palmar Hyperhidrosis: A Proposed Technique to Overcome Compensatory Hyperhidrosis and Improve Plantar Hyperhidrosis. Journal of Laparoendoscopic \& Advanced Surgical Techniques , 2,370-374

21. Callejas, M. A., Grimalt, R., Cladellas, E. (2010). Hyperhidrosis Update. Actas Dermosifiliogr, 101,110-118. 